\section{Dr. Carlos Casar Collazo (1940-2018)}

El doctor Carlos Casar Collazo nació en Santiago en 1940. Hijo de padres españoles. Su educación universitaria la realizó en la Universidad Católica y recibió el título de médico cirujano en 1965. Inició su carrera como "Médico General de Zona", contrato estatal con sede rural, que aseguraba beca de especialización en la Universidad de Chile. El eligió Curanilahue, 18.000 habitantes, 85 kilómetros al sur de Concepción, provincia de Arauco. Llegó a este destino en 1965. En esos años Curanilahue estaba bastante aislado, para ir en automóvil desde Concepción el camino era interrumpido por un tramo perteneciente a una de las primeras compañías forestales instaladas en la región debiendo, incluso las ambulancias, solicitar la apertura de una puerta con llave. En un ambiente de gran pobreza, analfabetismo y subdesarrollo el trabajo era intenso y agobiador. Por la ausencia de viviendas apropiadas los médicos residían en casas proporcionadas por el Hospital. Además de la atención en horas hábiles, con gran frecuencia se presentaban emergencias a todas horas. Aún con este trabajo los jóvenes médicos lograron concretar iniciativas como una Central de Diagnóstico y Tratamiento Controlado de la Tuberculosis y ciclos de Educación Sanitaria a grupos de madres y trabajadores.

Conocí a Carlos cuando llegó al Hospital Roberto del Río a efectuar su Beca de Especialidad en Medicina Infantil en el Departamento de Pediatría dirigido por el Profesor Julio Meneghello. La beca consistía en 3 años de alta exigencia académica y laboral, con atención directa de pacientes hospitalizados o concurrentes al consultorio adosado y turnos de residencia diurnos y nocturnos. Además, el médico becado debía tomar parte protagónica en todas las actividades académicas que prestigiaban la docencia y la investigación en ese establecimiento.

Terminada la beca ingresó al Laboratorio Funcional Respiratorio, iniciado poco antes por la Dra. Eliana Ceruti. Allí Eliana, Carlos y yo trabajamos a jornada completa en la atención de pacientes del Consultorio Adosado, realizábamos los exámenes funcionales, las interconsultas en sala y el seguimiento de pacientes con enfermedades respiratorias en la Unidad de Infecciosos.

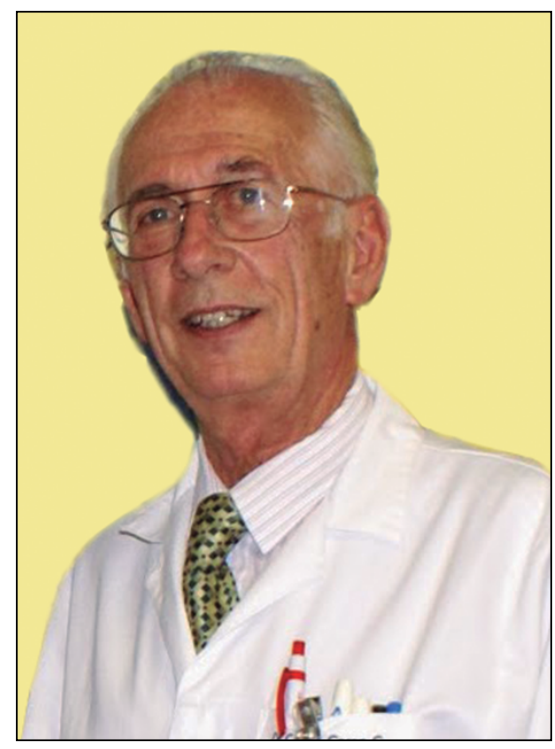

Con la finalidad de facilitar el trabajo se fue concentrando instrumental clínico y personal más entrenado en atención de pacientes respiratorios complejos: este fue el germen de la Unidad de Cuidados Especiales, posteriormente Unidad de Cuidados Intensivos. En esas salas se efectuaban nebulizaciones, punciones y drenajes pleurales, kinesioterapia respiratoria, endoscopía bronquial e incluso respiración artificial, todo con la valiosa participación de los becados residentes. Así funcionó varios años la Unidad de Cuidados Especiales hasta lograr la implantación de un sistema propio de residentes, pasando a ser en propiedad Unidad de Tratamiento Intensivo. Los tres médicos "broncopulmonares" vivimos esa evolución, alternándonos períodos de jefatura.

En la etapa inicial del Laboratorio llegaron equipos vistos por primera vez en Chile, en una época de escasa o ninguna asesoría técnica, debiendo asumir su montaje y puesta en función. Así con entusiasmo y empuje la Dra. Ceruti montó e hizo funcionar el equipo Radiometer de determinación de gases en sangre y equilibrio ácido-base, que pronto se hizo indispensable en el hospital. Al llegar Carlos demostró, además de sus capacidades y espíritu de trabajo y estudio, una gran habilidad manual colaborando en la instalación del Espirómetro Collins de 9 L. Además se instaló la determinación de volúmenes pulmonares con el espirómetro de Tissot y de medición de gases en sangre. También, sin apoyo técnico, se recibió el primer Ventilador Artificial Infantil de Presión Positiva, el pequeño Bird, que funcionaba sin electricidad, solo con la presión del aire comprimido u oxígeno y que a pesar de 
su incipiente sistema de control de parámetros permitió el manejo clínico de pacientes, gracias a que recién se disponía de la medición de gases en sangre arterial.

En ese tiempo los especialistas respiratorios trabajábamos en tres frentes: Consultorio Adosado, Laboratorio Funcional y Unidad de Cuidados Especiales. Posteriormente Carlos asumió con dedicación esta última labor; además ingresó a la Sociedad de Medicina Intensiva y realizó varias publicaciones al respecto.

Un recuerdo simpático, ahora histórico, es lo siguiente: en los primeros años del Laboratorio (1970-1975) no se disponía en Chile de calculadoras electrónicas ni sistemas informáticos o computacionales. El registro del espirómetro era mecánico: una campana sellada bajo agua que recibía los volúmenes de aire y transmitía sus desplazamientos a una pluma con tinta que inscribía un trazado real sobre un gráfico en papel calibrado en $\mathrm{ml}$ adosado a un tambor que giraba a una velocidad establecida por un mecanismo de relojería (kimógrafo). En la curva trazada en el papel calibrado en ml se marcaban manualmente puntos que medían los volúmenes pulmonares. La capacidad funcional pulmonar se calculaba a partir de las concentraciones de gases en el aire espirado al Tissot. El nitrógeno se medía con un Nitranalyzer. Los cálculos correspondientes se hacían con regla de cálculo. Por no disponer de computadores en los trabajos de investigación, la integración de los datos se hacía con máquinas calculadoras mecánicas y los cálculos estadísticos aplicando fórmulas algebraicas. Todo esto significaba un enorme requerimiento de tiempo. La aplicación de métodos electrónicos y computacionales pronto permitió medir rápida y fácilmente los parámetros nombrados y muchos otros. Los actuales equipos computarizados de estudio funcional respiratorio proporcionan todos los datos necesarios en pocos instantes, incluso ya comparados con valores normativos predichos. Los procedimientos "manuales" ya recordados mantienen un gran valor metódico y conceptual. El espirómetro Collins se considera un instrumento de precisión y constituye un verdadero "Gold Standard" para los equipos actuales. Desde un inicio el doctor Casar fue pionero en el estudio y aplicación de la informática y en promover su difusión.

Otro aspecto de la especialidad que asumió con su habitual entusiasmo y eficacia fue la Tuberculosis infantil. Debió organizar la recepción de interconsultas y el cumplimiento del Programa Nacional de Control y Erradicación de la Tuberculosis en todos los consultorios periféricos, ar- dua misión que pudo realizar con la colaboración entusiasta de las enfermeras universitarias y el personal paramédico. En la pesquisa de contactos más de una vez debieron efectuar investigaciones francamente detectivescas. Participaba en las sesiones del Comité Ministerial y también como asesor viajaba a otras áreas o a provincias. La experiencia lograda en el tema le permitió escribir el capítulo de Tuberculosis en el texto de Pediatría del Profesor Meneghello y colaborar en el libro "Tuberculosis" del Profesor Victorino Farga.

En el trabajo académico Carlos participaba a diario en la docencia de alumnos, internos de Medicina y Becados de Post grado. Con los becados el trabajo conjunto en los turnos de residencia le significó la más alta estima de estos médicos agradecidos. Participaba en los cursos y seminarios que organizaba el Departamento y asistió a innumerables congresos dentro y fuera de Chile, lo que lo hizo ampliamente conocido y respetado. Publicó gran número de trabajos científicos en revistas chilenas y extranjeras y colaboró permanentemente en las Normas de Atención Pediátrica del Hospital. Con el tema Tuberculosis colaboró en el texto "Pediatría" del Profesor Meneghello. En 1970 ingresó a la Sociedad de Pediatría y poco después a la Sociedad de Enfermedades Respiratorias. Fue presidente de la Rama de Enfermedades Respiratorias de la Sociedad de Pediatría en los años 1973 y 1974. Formó parte del directorio de la Sociedad de Enfermedades Respiratorias en los años 1982 y 1983. Presidió el Comité de Tratamiento Intensivo de la Sociedad de Pediatría. Fue Subjefe del Servicio de Pediatría en los años 1989 a 1990 y 1994 a 2000. Se desempeñó como Jefe de Servicio en los años 2000 a 2002. La Universidad de Chile lo nombró Profesor Adjunto de Pediatría y la Sociedad Chilena de Enfermedades Respiratorias lo designó Miembro Honorario.

"La muerte no es tan verdadera cuando se ha cumplido decididamente bien la obra de la vida". Esta afirmación del poeta José Martí la estamos constatando todos los que tuvimos el privilegio de conocer a Carlos Casar. El dejó evidentemente una impronta indeleble. Impronta para la Real Academia Española: "marca o huella que en el orden moral deja un individuo o suceso".

"Siempre contento". Esta era su característica personal más importante. Contento: alegría íntima que en Carlos trascendía a la persona y se debía al sentimiento generoso de cumplir metas auto propuestas: el bienestar y mejoría de los pacientes y un aprendizaje marcador en los alumnos, todo en un ambiente de solidaridad. Cuidadoso y cariñoso en la atención de los ni- 
ños, respetuoso pero al mismo tiempo con buen humor, hacía comentarios e incluso bromas, con lo que lograba una comunicación empática que facilitaba exploraciones molestas como la observación de las amígdalas o colocación de vacunas y terminaba todo en grandes manifestaciones de amistad de los niños y sus padres. De carácter calmado, nunca se apresuraba en su trabajo, decía: "estaré atrasado, pero jamás apurado".

A su lado durante 50 años estuvo su esposa Maricarmen Leturia, Licenciada en Biología y Educación para la Salud, también hija de españoles. Ella lo acompañó desde Curanilahue, donde colaboró en el trabajo hospitalario y acciones comunitarias y además solucionó la carencia de profesores en el Liceo local, dictando clases de Biología y Química. Posteriormente su principal preocupación fue la atención de sus hijos, 3 niños y 2 niñas. A ellos les reiteramos nuestro apoyo incondicional en el recuerdo imperecedero de este amigo inolvidable, y para ti Carlos esto no es un "Adiós", sino un "Gracias".

Dr. Armando Díaz Cruz Sociedad Chilena de Enfermedades Respiratorias 